\title{
HISTORIA
}

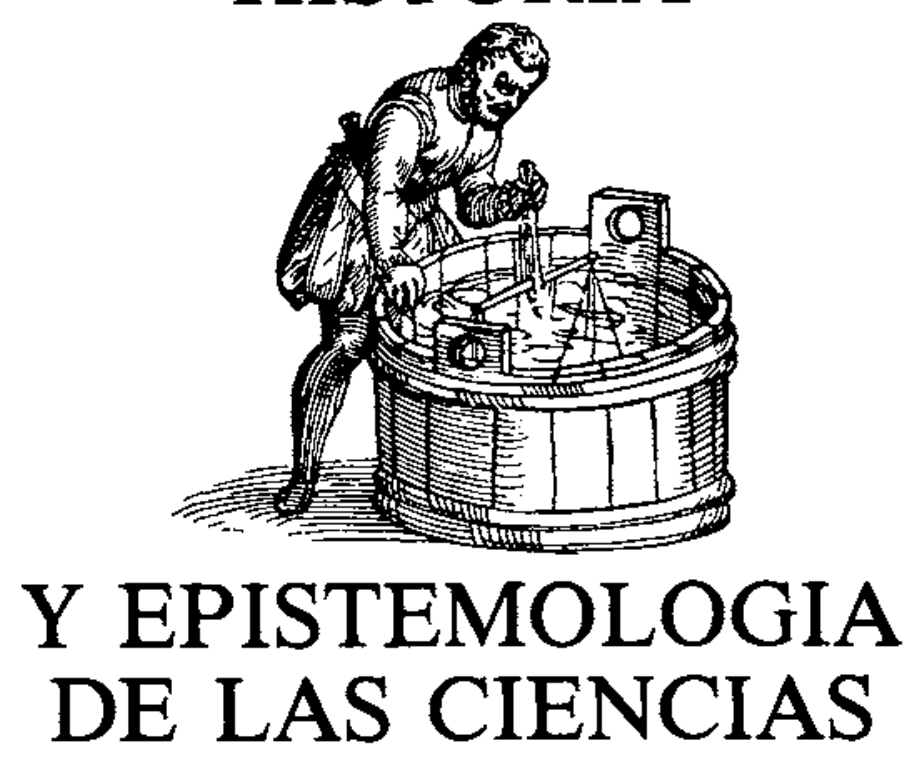

\section{CONTRIBUCIÓN DE LA HISTORIA Y DE LA FILOSOFÍA DE LAS CIENCIAS AL DESARROLLO DE UN MODELO DE ENSEÑANZA/APRENDIZAJE COMO INVESTIGACIÓN'}

GIL PÉREZ, D.

Universitat de València.

\section{SUMMARY}

After the fiasco of the «leaming by discovery» orientation and the serious limitations of the "reception learning" paradigm, a new consensus is emerging on the nature of mathematics and science learning as a construction of knowledge. These constructivist approaches have taken explicitly into account contemporary views in philosophy of science as an epistemological base for a conception of learning as a conceptual change. Teaching strategies oriented to produce conceptual changes have been developed with promising results, but serious difficulties have also appeared. Our contribution will try to show that those difficulties are due to a still insufficient consideration of the nature of science in the teaching strategies. Consequently, we shall propose some modifications of these strategies, taking more carefully into account the relationship between the nature of science and the nature of learning.

\section{INTRODUCCIÓN}

En la orientación đe la enseñanza de las ciencias, la psicología -y más concretamente la psicología educati-

va-- ha jugado un papel esencial, como revelan las numerosas referencias que en este campo se hacen a 
Ausubel, Bruner, Dewey, Gagné, Piaget, etc. Se trata, sin duda, de una influencia lógica a la que se deben, además, notables contribuciones como la crítica a la enseñanza por descubrimiento autónomo (Ausubel 1978) - la emergencia de Ias orientaciones constructivistas (Coll 1988). Muy a menudo, sin embargo, esta relación entre la enseñanza de las ciencias y la psicología se ha planteado como mera aplicación externa, de acuerdo con una concepción de la formación docente como suma de una preparación científica básica y una formación psicosocio-pedagógica general desconectada de las disciplinas concretas, cuyas insuficiencias han sido reiteradamente puestas de relieve (Gil 1982a, McDermott 1990). Pero, afortunadamente, el desarrollo de la didáctica de las ciencias como dominio específico ha permitido, por una parte, integrar de manera más eficaz las aportaciones de las ciencias de la educación y, por otra, ampliar su fundamentación considerando las posibles aportaciones de la historia y filosofía de la ciencia en la enseñanza de las ciencias (Izquierdo 1992). El objeto de este trabajo consiste, precisamente, en poner de relieve el papel de la historia y de la fílosofía de la ciencia en la enseñanza de las ciencias. Con ello no nos estamos refiriendo simplemente al reconocimiento del papel educativo que estas disciplinas pueden tener y que ha sido repetidamente resaltado por científicos, historiadores de la ciencia y educadores (Langevin 1926, Navarro 1983, Moreno 1986, Sánchez Ron 1988, Matthews 1990...). Nos referimos más espećfficamente al papel que la historia y filosofía de las ciencias pueden jugar en el desarrollo de un cuerpo específico de conocimientos didácticos. Las aportaciones en este campo han sido relativamente escasas hasta muy recientemente, pero el desarrollo de la didáctica de las ciencias ha terminado por impulsar una potente línea de investigación centrada en las relaciones entre historia y filosofía de las ciencias y la enseñanza de las ciencias. La creciente fecundidad de este campo de investigación se evidencia en los números monográficos publicados recientemente en revistas como International Journal of Science Education, Journal of Research in Science Teaching o Science Education, en la celebración de numerosos congresos internacionales o en el surgimiento en 1992 de la revista específica Science and Education.

También en nuestro país, la atención a la historia y filosofía de la ciencia desde el campo de la didáctica se ha đesarrollado estos últimos años. Un claro índice de elio es, por ejemplo, la sección «Historia y epistemología de las Ciencias» de la revista Enseñanza de las Ciencias, en la que se ha publicado numerosos artículos de interés desde su aparición en 1983.

Nos encontramos, pues, en un momento en que la Didáctica de las Ciencias ha empezado a considerar con atención las aportaciones potenciales de la historia y filosofía de las ciencias en Ia enseñanza de las ciencias, y a utilizarlas explícitamente en Ia fundamentación de sus propuestas. Este trabajo pretende ser una revisión de lo que la renovación de la enseñanza de las ciencias debe ya a la historia y filosofía de las ciencias y que podemos sintetizar en la idea de una creciente aproximación de las situaciones de aprendizaje a las de una actividad científica. Ello supone, en primer lugar, reinterpretar lo que significó el movimiento de renovación asociado al denominado «aprendizaje por descubrimiento» y romper con el rechazo que dicho movimiento generó a la asociación entre aprendizaje de las ciencias y trabajo científico. En efecto, se afirma habitualmente que ei modelo de aprendizaje por descubrimiento, que se desarroll $\delta$ y llevó a la practica durante las décadas de los 60 y 70 , constituy 6 un intento de aproximar el aprendizaje de las ciencias a las características del trabajo científico: «Se pretendió que Ios niños disfrutaran con la ciencia (mediante una implicacín más directa en actividades científicas), ađquirieran una visión más clara de lo que hacen los científicos y fueran impulsados a proseguir estudios científicos de nivel superior" (Hodson 1988). Y dado que dicha orientación se saldó, según todos los análisis, con un grave fracaso (Ausubel 1968, Gil 1983, Hodson 1985, Millar y Driver $1987 \ldots)$, se podría pensar que resulta inadecuado pretender que los alumnos se impliquen en actividades científicas o incluir entre los objetivos de la educación la familiarización con las caracterísiticas đel trabajo científico.

Intentaremos mostrar, sin embargo, que la idea de aproximar el aprendizaje de las ciencias a la actividad científica no se circunscribe al modelo de aprendizaje por descubrimiento, sino que constituye un auténtico hilo conductor -aunque no siempre explícito-en la transformación de la enseñanza de las ciencias. Un hilo conductor que se ha mostrado fructífero incluso a través de los errores cometidos y que se ve reforzado tanto por las orientaciones constructivistas que constituyen hoy el paradigma emergente (Resnick 1983, Novak 1988, Wheatley 1991...) como por las implicaciones de la epistemología contemporánea en la enseñanza (Posnet et al. 1982, Gil y Carrascosa 1985 y 1990 , Hodson 1988, Matthews 1990, Cleminson 1990, Cobb, Wood y Jackel 1991, Duschl y Gitomer 1991, Gruender y Tobin 1991, Sequeira y Leite 1991...). En lo que sigue, analizaremos, desde esta perspectiva, las grandes tendencias innovadoràs en la enseñanza de las ciencias.

\section{EL MODELO DE APRENDIZAJE POR DESCUBRIMIENTO: ALGO MAS QUE UN FRACASO}

No vamos a insistir aquí en los abundantes análisis que han mostrado con toda claridad el fracaso del paradigma de aprendizaje por descubrimiento. El inductivismo extremo en que dicho modelo incurría, la falta de atencion a los contenidos, la insistencia en una actividad comple tamente autónoma de los alumnos, etc. tienen poco que ver, en efecto, con la visión actuâl de lo que constituye el trabajo científico y se ha mostrado con claridad el resultado negativo de su aplicación, tanto en lo que se refiere a la adquisición de conocimientos como en lo que respecta a la comprensión de la naturaleza de la ciencia (Ausubel 1968, Gil 1983, Hodson 1985, Millar y Driver 1987...). Consideramos, sin embargo; que este análisis crítico, aunque certero, resulta parcial y no debe traducirse en un rechazo global, sino que se precisa un análisis más matizado de lo que supuso este amplio movimiento de reforma. 
En primer lugar podemos señalar que la idea de convertir la educación científica -en su sentido más amplio de familiarización con los logros fundamentales de las ciencias y con sus métodos-en una componente esencial de la formación básica de los futuros ciudadanos constituye una intuición básica asociada a nombres de relevantes científicos y educadores como Dewey, Langevin o Piaget.

La corriente de aprendizaje por descubrimiento intento desarrollar plenamente la idea de que los estudiantes debían familiarizarse con las actividades del trabajo científico para poder comprender los conocimientos alcanzados. Con esta implicación de los alumnos en actividades científicas, se pretendía proporcionar una visión abierta y accesible de la ciencia, favoreciendo una actitud más positiva hacia la misma, así como llamar explícitamente la atención sobre la especificidad y efectividad de sus métodos. Y, si bien es cierto que los resultados alcanzados distaron mucho de los objetivos perseguidos, la orientación de aprendizaje por descubrimiento supuso un intento sistemático de renovación curricular que rompía con una estabilidad de muchas décadas e iniciaba un proceso de transformación en el que seguimos hoy inmersos, sin que los objetivos de aproximar la actividad de los alumnos a las caracterísiticas del trabajo científico y, sobre todo, de generar actitudes positivas hacia la ciencia y su aprendizaje, hayan perdido su vigencia. Muy al contrario, como indica, por ejemplo, Solomon (1991), «para la mayoría de los alumnos, nuestros objetivos básicos pueden consistir en generar actitudes favorables hacia la ciencia y una cierta comprensión por sus métodos de construcción de teorías». Y más contundentemente aún, Burbules y Linn (1991) afirman: «Una forma de plantear el objetivo central de la educación científica es preguntarnos cuál es la actitud epistemológica que queremos propiciar en los estudiantes.»

Desde ese punto de vista, los resultados conseguidos por el paradigma de aprendizaje por descubrimiento no pueden interpretarse simplemente como un fracaso, sino como el origen de las reestructuraciones posteriores, como un elemento dinamizador de una enseñanza que permanecía anclada en tradiciones asumidas acríticamente. De hecho, los resultados negativos alcanzados dirigieron Ia atención hacia las concepciones subyacentes acerca de la ciencia, iniciándose una revisión crítica đe las mismas (Leboutet 1973, Host 1978, Giordan 1978) y una aproximación a las adquisiciones de la filosofía contem. poránea de la ciencia. De este modo, el modelo de aprendizaje por recepción significativa (Ausubel 1968, Novak 1979) que se desarrolló como reacción frente al de descubrimiento, aunque puede interpretarse, en cierto modo, como un retomo a las formas más tradicionales de enseñanza por simple transmisión de conocimientos ya elaborados -e incluso como un rechazo explícito de la asociación aprendizaje de las ciencias / familiarización con las características del trabajo científico-supuso, en algunos aspectos, una más correcta aproximación a la naturaleza de la ciencia.
EL MODELO DE APRENDIZAJE POR RECEPCION SIGNIFICATIVA: ¿UNA RUPTURA TOTAL CON LA IDEA DE APROXIMAR EL APRENDIZAJE A LAS CARACTERISTICAS DEL TRABAJO CIENTIFICO?

Como acabamos de señalar en el apartado anterior, la crítica de la enseñanza por descubrimiento inductivo y autónomo vino acompañada de la defensa del aprendizaje por recepción o, dicho de otro modo, de la enseñanza por transmisión. Ello no supuso, sin embargo, un simple retorno al modelo «tradicional» y consiguiente retroceso en la renovación de la enseñanza, ni siquiera puede interpretarse como una completa desviación del objetivo fundamental de familiarizar a los alumnos con la naturaleza del trabajo científico, aunque una primera lectura así parezca indicarlo.

Es cierto, en efecto, que Ausubel (1968) fundamenta la defensa de la enseñanza por transmisión, entre otros, en la "falta de capacidad de la mayoría de los alumnos para descubrir autónomamente todo lo que deben saber» y que ello puede interpretarse como un rechazo de la asociación del aprendizaje de las ciencias con la familíarización con la metodología científica. Sin embargo, una consideración atenta de algunas propuestas de Au* subel o Novak muestran una indudable coherencia con tesis básicas de la epistemología contemporánea acerca de la naturaleza de la ciencia. Así, por ejemplo, la importancia concedida por Ausubel a los conocimientos previos de los alumnos y a la integración de los nuevos conocimientos en sus estructuras conceptuales es coherente con el papel que las concepciones de los científicos, es decir, los paradigmas teóricos, juegan en todo el proceso de investigación científica. Del mismo modo, al resaltar el papel que la guía del profesor puede jugar como facilitadora de un aprendizaje significativo -en vez de Ias adquisiciones dispersas que proporcionan los «des" cubrimientos" incidentales del trabajo autonomo-, Ausubel se aproxima a una característica básica del trabajo científico: cualquier investigador novel conoce bien el papel que juegan quienes dirigen su investigación o las discusiones con sus compañeros más experimentados, lo que en ningún caso permite hablar de trabajo autonomo o de descubrimiento incidental.

En aspectos como los señalados, el modelo de aprendi* zaje por recepción significativa está, sin duda, más cerca de la naturaleza del trabajo científico que el del aprendizaje por descubrimiento. Se trata, claro está, de una aproximación insuficiente que entra, además, en colisión con otras características básicas de la metodología científica. Pero conviene resaltar, como hemos hecho, que algunos aspectos particularmente válidos de este paradigma resultan coherentes con la idea de aproximar la actividad de los alumnos a la construcción de conocimientos científicos. Las contradicciones del modelo con to que supone la actividad científica son, sin embargo, muy evidentes, puesto que explícitamente plantea la asimilación de conceptos, renunciando a que los alumnos participen en su construcción. De este modo el papel de guía del profesor se convierte en proporcionar los conocimientos ya elaborados para su aprendizaje por 
recepción. Y si bien es cierto, como señala Ausubel, que la verdadera asimilación de conceptos exige un proceso activo de relación, diferenciación y reconciliación integradora con los conceptos pertinentes que ya existían, no está nada claro que una enseñanza por transmisión de conocimientos «en su estado final" favorezca dicha actividad. Ello exigiría, al menos, tener en cuenta las necesidades de tiempo propio para que los alumnos puedan trabajar los conceptos hasta ligarlos a su estructura conceptual. Y habría que plantear las actividades que favorezcan dicho trabajo de relación, diferenciación... e introducir los mecanismos de retroalimentación para constatar hasta qué punto los alumnos han asimilado y se puede o no seguir adelante.

En definitiva, hacer activo el proceso de asimilación en la clase supondría más trabajo (dirigido) de los alumnos y más tiempo propio para éstos, aproximando así la asimilación a la construcción de conocimientos (Gil 1983). Por otra parte, aun cuando el modelo de aprendizaje por recepción significativa rechaza el inductivismo característico del aprendizaje por descubrimiento incidental y autónomo, conviene notar que tras las propuestas de asimilación de un conocimiento ya elaborado persisten las tesis inductivistas. En efecto, los conceptos siguen considerándose algo externo al sujeto que debe ser «captado» por éste.

El aprendizaje por recepción se aleja así de las concepciones actuales acerca de cómo se construyen los conocimientos científicos, y cabría atribuir a dicho alejamiento -ésa es, al menos, nuestra hipótesis- su probada inefectividad para lograr los modestos objetivos de un aprendizaje exclusivamente conceptual que deja de lado los aspectos procedimentales y axiológicos. En efecto, las investigaciones sobre "errores conceptuales» cuestionaron de forma radical la efectividad de este modelo de transmisión/recepción de conocimientos ya elaborados. Por otra parte, aunque el modelo preste atención exclusivamente a los aspectos conceptuales, no por ello deja de transmitir una determinada imagen de lo que es la ciencia y de cómo se forman los conocimientos científicos,implícita en el propio currículo (Hodson 1985, King 1991). De esta forma, el modelo de aprendizaje por recepción no sollo se mostraba incapaz de lograr una apropiación de los conceptos realmente significativa, sino que contribuía a las visiones deformadas y empobrecidas que los alumnos adquieren sobre el trabajo científico y que son responsables, en gran medida, de las actitudes negativas hacia la ciencia y su aprendizaje (Schibecci 1984, Escudero 1985, James y Smith 1985, Yager y Penick 1986...).

La necesidad de un replanteamiento en profundidad del proceso de enseñanza/aprendizaje de las ciencias se imponía así de nuevo. Los resultados proporcionados por los modelos de aprendizaje por descubrimiento y por recepción significativa no habrán supuesto, sin embargo, simples pérdidas de tiempo y esfuerzos. Ahora se tenía una visión más clara de las dificultades y de la necesidad de planteamientos más rigurosos, sin caer en descalificaciones simplistas de una "enseñanza tradicional» fácilmente superable. Y el esfuerzo de fundamentación iba a dirigir de nuevo la atención hacia las analogías entre la actividad de los científicos y la de los alumnos, haciendo converger los hallazgos de la psícología del aprendizaje con las aportaciones de la filosofía e historia de las ciencias contemporáneas.

\section{LA ORIENTACIÓN CONSTRUCTIVISTA Y LAPROFUNDIZACIÓNENLA NATURALEZA DEL TRABAJO CIENTIFICO COMO LOS AYANCES (CONVERGENTES) MÁS FRUC. TIFEROS DE ESTAS ULTIMAS DECADAS EN LA ENSENANZA/APRENDIZAJE DELAS CIENCIAS}

No vamos a insistir aquí en la importancia de la orientación constructivista que constituye, sin duda, el consenso emergente en la enseñanza de las ciencias (Resnick 1983), y que ha sido calificada como la aportación más relevante de las últimas décadas en este campo (Gruender y Tobin 1991). Tampoco creemos necesario extendernos en la descripción de las características esenciales de la nueva visión del aprendizaje, expuestas con detalle por diversos autores (Posner et al. 1982, Driver 1986...). Resnick los resume en tres principios (Resnick 1983):

- Quienes aprenden construyen significados. No reproducen simplemente lo que leen o lo que se les enseña.

- Comprender algo supone establecer relaciones (...) Los fragmentos de información aislados son olvidados o resultan inaccesibles a la memoria.

- Todo aprendizaje depende de conocimientos previos.

Se trata, como señala la autora, de una simplificación que esconde muchas complejidades, pero permite apreciar ya una indudable similitud con tesis básicas acerca de cómo se construyen los conocimientos científicos (Kuhn 1971). Esta similitud ha sido puesta de relieve por numerosos autores (Posner et al. 1982, Gil 1983, Gil y Carrascosa 1985 y 1990, Burbules y Linn 1991, Cobb, Wood y Yackel 1991, Duschl y Gitomer 1991...) y aparece como uno de los apoyos fundamentales de la nueva orientación. De hecho, las propuestas constructivistas han mostrado una gran capacidad integradora de estudios muy diversos: desde la epistemología contemporánea (Bachelard, Kuhn, Lakatos, Toulmin, Feyerabend...) a las concepciones constructivistas de Kelly, pasando por los trabajos de Piaget o Vigotskii. Esta coherencia básica de los resultados de investigaciones inicialmente inconexas ha reforzado, sin duda, el valor de las concepciones constructivistas sobre la enseñanza/aprendizaje de las ciencias y ha permitido la emergencia de un amplio consenso.

El viejo objetivode aproximar las actividades de aprendizaje a las de la construcción de los conocimientos científicos ha cobrado así nueva fuerza, apoyado en una mejor comprensión de la naturaleza de la ciencia y en una sólida fundamentación teórica. Queremos insistir, sin embargo, en que este avance ha sido posible gracias, en parte, a los intentos precedentes, con sus «errores»y consiguientes reorientaciones. Con demasiada frecuen- 
cia se olvida esta relación entre los distintos modelos contrapuestos, con lo que el desarrollo de la didáctica de las ciencias parece sujeto a una azarosa agitación. Si se desea reforzar el carácter de cuerpo específico de conocimientos (en construcción) de la didáctica de las ciencias y superar los tratamientos ateóricos, es preciso tener en cuenta las aportaciones de los distintos modelos, su capacidad para resistir a las modificaciones (lo que es índice de una cierta coherencia y efectividad) y, en particular, resaltar su contribución al surgimiento de nuevas orientaciones (en ocasiones a través de la constatación de sus insuficiencias).

Igualmente resulta necesario reconocer la coincidencia básica-al margen, obviamente, de algunos matices-de diversos propuestas presentadas por sus autores como modelos distintos. Esto es lo que está ocurriendo hoy con las estrategias de enseñanza de orientación constructivista presentadas por diferentes autores (Nusbaum y Novick 1982, Posner et al. 1982, Osborne y Wittrock 1983 y 1985, Driver y Oldham 1986, Hewson y Hewson 1988 , Hodson 1988, Giordan 1989, Pozo 1989...). En todas ellas encontramos, expresadas en una u otra forma, la idea de contemplar el aprendizaje como un cambio conceptual que precisa de:

- una fase de elicitación de las concepciones de los alumnos (haciendo aparecer el carácter plausible y fructífero que las mismas poseen en los contextos utilizados por los alumnos);

- una fase đe reestructuración, con la creación de conflictos cognitivos que generen la insatisfacción con dichas concepciones y preparen para la introducción de los conceptos científicos; y

- una fase de aplicación que proporcione oportunidades a los alumnos para usar las nuevas ideas en diferentes contextos.

El cuadro I esquematiza dichas estrategias. El esquema posee una aparente lógica y ha sido seriamente fundamentado por distintos autores hasta convertirse, con ligeros matices diferenciadores, en la estrategia de enseñanza que podemos denominar como cambio conceptual. Una estrategia cuyos elementos básicos y diferenciadores resultan ser la identificación de las ideas que ya poseen los alumnos y la creación de los conflictos cognoscitivos que generen la insastifacción de los alumnos con dichas ideas.

La efectividad de estas estrategias de cambio conceptual viene refrendada por numerosas investigaciones realizadas en diferentes campos de las ciencias (Nusbaum y Novick 1982, Anderson y Smith 1983, Hewson y Hewson 1984, Ministrell 1984, Roth 1984, Osborne y Freyberg 1985, Zietsman y Hewson $1986 \ldots$..).

Otros autores, sin embargo, han constatado que ciertas concepciones alternativas son resistentes a la instrucción, incluso cuando ésta se orienta explécitamente a producir el cambio conceptual (Fredette y Lochhead 1981, Engel y Driver 1986, Shuell 1987, White y Gunstone 1989, Duschl y Gitomer 1991...).
Cuadro I

Estrategias de enseñanza para un aprendizaje como cambio conceptual.

1. Identificación y clarificacion de las ideas que ya poseen los alumnos.

2. Puesta en cuestion de las ideas de los estudiantes, a través del uso de contraejemplos (conflictos cognoscitivos).

3. Introducción de nuevas ideas, mediante brainstorming, entre los alumnos o presentadas por el profesor.

4. Proporcionar oportunidades a los alumnos para usar las nuevas ideas en diferentes contextos.

Estas dificultades y la preocupación por afianzar la fundamentación del modelo obligan, en nuestra opinión, a un análisis en profundidad de las estrategias de ensefianza propuestas. Veremos esto en el próximo apartado.

\section{CAMBIO CONCEPTUAL VERSUS CAMBIO CONCEPTUAL Y METODOLOGICO}

Una primera y, a nuestro entender, seria limitación de las propuestas de «cambio conceptual», a la que venimos haciendo referencia desde principios de la década de los 80 , es la falta de suficiente atención a las formas de razonamiento asociadas a los esquemas alternativos de los alumnos (Carrascosa y Gil 1982, Gil y Carrascosa 1985 y 1990, Gil, Carrascosa, Furió y Martínez-Torregrosa 1991). Sintetizaremos los argumentos que hemos expuesto extensamente en dichos trabajos:

- Existe una cierta semejanza entre los esquemas alternativos de los alumnos en algunos campos y concepciones históricas que fueron desplazadas por los conocimientos hoy aceptados por la comunidad científica (Piaget 1970, Champagne et al. 1980, Disessa 1982, Clement 1983, McDermott 1984, Saltiel y Viennot 1985, Furió et al. 1987, Mathews 1990).

- Sin intentar establecer un paralelismo mecánico entre Ias concepciones alternativas de los alumnos y las precientíficas, parece razonable suponer que dicha semejanza no puede ser accidental, sino el resultado de una forma también similar de abordar los problemas (Gil y Carrascosa 1985). Esta hipótesis se ve reforzada por el estudio comparado de las características de lo que puede denominarse «Física del sentido común» (Bachelard 1938, Holton y Roller 1963, Koyre 1981) y de las formas de razonamiento de los alumnos. Se constata, en efecto, una misma estrategia de aproximación a los problemas, que hemos denominado «metodología de la superficialidad» (Carrascosa y Gil 1985) o del sentido común 
(Hashweh 1986); caracterizada por la ausencia de dudas o consideración de posibles soluciones alternativas, por respuestas rápidas y seguras basadas en las evidencias del sentido común, por tratamientos puntuales, sin búsqueda de una coherencia global en el análisis de diferentes situaciones (Ministrell 1982, Whitaker 1983, Halloun y Hestener 1985, Hewson 1985, Champagne, Gunstone y Klopfer 1985, Hewson y Torley $1989 .$. ), por razonamientos que siguen una secuencia causal lineal (Closset 1983, Viennot 1989), etc.

- Las concepciones preclásicas sólo pudieron ser desplazadas gracias a una nueva metodología que combinaba la creatividad del pensamiento divergente con el rigor en la contrastación de las hipótesis, mediante experimentos en condiciones controladas y la búsqueda de coherencia global. Cabe, pues, pensar que los cambios conceptuales de los alumnos exijan igualmente un profundo cambio metodológico o, si se prefiere, epistemológico (Gil y Carrascosa 1985 y 1990, Hashweh 1986, Cleminson 1990, Duschl y Gitomer 1991), puesto que se trata de «fundamentar un conocimiento contra el pensamiento común» (Agabra, Astolfi et al. 1985)

Históricamente, ese cambio a la vez conceptual y metodológico no fue en absoluto fácil y es iógico pensar que lo mismo ocurrirá con los estudiantes: solamente, si son puestos reiteradamente en situación de apropiarse la nueva metodología (por ejemplo, en situación de construir hipótesis, diseñar experimentos, realizarlos y analizar cuidadosamente los resultados, con una atención particular a la coherencia global, etc.), será posible que superen su metodología espontánea de sentido común, al tiempo que se producen los profundos cambios metodológicos que exige la construcción del conocimiento científico. Como señalan Astolfi, Verin y otros (1985): «Las nociones y los métodos científicos no se adquieren en una o dos veces mediante algunos ejemplos (demasiado) bien elejidos, sino que se logra apropiarlos lentamente, tras refuerzos y regresiones aparentes.»

Las consideraciones anteriores implican una primera crítica de las estrategias de enseñanza orientađas a producir cambios conceptuales. En efecto, dichas estrategias parecen poner casi exclusivamente el acento en la modificación de las ideas. $Y$ aunque es cierto, como señalan Hewson y Torley (1989), que el cambio conceptual tiene sus exigencias epistemológicas y no debe considerarse como un simple cambio del contenido de las concepciones, en nuestra opinión, es necesaria una mayor insistencia en que el cambio conceptual comporta un cambio metodológico, por lo que las estrategias de enseñanza han de incluir explícitamente actividades que asocien el cambio conceptual con la práctica de la metodología científica. Pensemos a este respecto que uno de los defectos de la enseñanza de las ciencias repetidamente denunciado ha sido el de estar centrada casi exclusivamente en los conocimientos declarativos (en los «que») olvidando los procedimentales (los «como»). No puede esperarse, pues, que baste hablar de cambio conceptual para que se tengan en cuenta las exigencias metodológicas y epistemologicas que ello comporta. Por el contrario, cabe temer que sin una insistencia muy explícita y fundamentada, las actividades creativas del trabajo científico - la invención de hipótesis, etc.- continuarán prácticamente ausentes de las clases de ciencias (Yager y Penick 1983, Gil y Martínez-Torregrosa 1984, Gil y Payá 1988, Hodson 1992). En el mismo sentido, Duschl y Gitomer (1991), profundizando en las implicaciones de la epistemología contemporánea, afirman: «si tenemos que producir una reestructuración radical de conceptos, lo que constituye el correlato personal de la idea kuhniana de revolución científica, parece que đeberíamos enseñar también los conocimientos procedimentales implicados». Duschl y Gitomer critican así, siguiendo a Laudan (1984), una visión jerárquica del cambio conceptual que asume que los cambios conceptuales producirán cambios simultáneos de procedimientos y actitudes. Y atribuyen a esta incorrecta apreciación la responsabilidad de la insuficiente atención prestada a los aspectos metodológicos y de la inefectividad parcial de las estrategias de cambio conceptual (Duschl y Gitomer 1991). Una vez más nos encontramos así en la necesidad de superar visiones epistemológicas incorrectas para hacer posible una transformación efectiva de la enseñanza de las ciencias. Sin embargo, aunque diversos autores han planteado, de forma más o menos directa, la cuestión de una relación entre los esquemas conceptuales alternativos y las formas de razonamiento o tratamiento de problemas asociados (Osborne y Wittrock 1983 y 1985 , Gil y Carrascosa 1985 y 1990 , Hashweh 1986, Reve et al. 1987 , Hills 1989, Viennot 1989, Burbules y Linn 1991...), las estrategias de enseñanza a que nos hemos referido en el apartado anterior, orientadas a provocar cambios conceptuales, no parecen incluir esta aproximación de la actividad de los alumnos a lo que constituye la investigación cientifica. De forma explícita, al menos, se pone el acento casi exclusivamente en las ideas que poseen los alumnos, lo que constituye, repetimos, una grave limitación. Pero aún es posible hacer otra crítica más importante, si cabe, a estas estrategias de enseñanza. Dedicaremos el próximo apartađo a esta cuestión.

\section{CAMBIO CONCEPTUAL VERSUS TRA. TAMIENTO CIENTIFICO DE SITUACIONES PROBLEMÁTICAS ABIERTAS}

La secuencia que proponen las estrategias de enseñanza basadas en el cambio conceptual consiste, como se recordará, en sacar a la Iuz las ideas de los alumnos, favoreciendo su formulación y consolidación, para después crear conflictos que las pongan en cuestión e introducir a continuación las concepciones científicas, cuya mayor potencia explicativa va a hacer posible el cambio conceptual. Es cierto que dicha estrategia puede, puntualmente, dar resultados muy positivos al llamar la atencion sobre el peso de ciertas ideas de sentido común, asumidas acríticamente como evidencias; pero también es cierto, que practicada de forma reiterada, produce una inhibicíon y un rechazo muy comprensibles. En efecto ¿qué sentido tiene hacer que los alumnos expliciten y afiancen sus ideas para seguidamente cuestionarlas? ¿Cómo no ver en ello un artificio que aleja la situación de lo que constituye la construcción de conocimientos? Esa cons- 
trucción nunca se plantea para cuestionar ideas, para provocar cambios conceptuales, sino para resolver problemas de interés para los investigadores (es decir, en nuestro caso para los alumnos, no únicamente para los profesores); problemas que se abordan, como es lógico, a partir de los conocimientos que se poseen y de nuevas ideas que se construyen a título tentativo. En ese proceso, las concepciones iniciales podrán experimentarcambios e incluso, aunque más raramente, ser cuestionadas radicalmente, pero ése no será nunca el objetivo, sino, repetimos, la resolución de los problemas planteados.

Desde un punto de vista constructivista resulta esencial asociar explícitamente la construcción de conocimientos a problemas - «Todo conocimiento es la respuesta a una cuestión" (Bachelard 1938) - y ello cuestiona de forma radical las estrategias de cambio conceptual en lo que supone tomar las ideas de los alumnos como punto de partida. Por otra parte, una característica fundamental del tratamiento científico de los problemas es tomar las ideas que se tienen -incluso las más seguras y obviascomo simples hipótesis de trabajo que es necesario controlar, esforzándose en imaginar otras hipótesis, etc. Ello concede un estatus muy diferente a las situaciones de conflicto cognoscitivo: ya no suponen para los alumnos el cuestionamiento externo de las ideas personales, ni la reiterada aceptación de las insuficiencias del propio pensamiento (con las consiguientes implicaciones afectivas), sino un trabajo de profundización en el que unas ideas (tomadas como hipótesis) son sustituidas por otras (tan propias como las anteriores).

No se trata, como puede verse, de eliminar los conflictos cognoscitivos, sino de evitar que adquieran el carácter de una confrontación entre las iđeas propias (incorrectas) y los conocimientos científicos (externos). A este respecto, Solomon (1991) argumenta que «tras impulsar la expresión de un conjunto de opiniones particulares, el profesor no puede simplemente rechazar las que no se ajustan a la teoría vigente. De ese modo dejaría de ser posible un diálogo abierto».

Por todo ello, la estrategia de enseñanza que nos parece más coherente con la orientación constructivista y con las características del razonamiento científico es la que plantea el aprendizaje como tratamiento de situaciones problematicas abiertas que los alumnos puedan considerar de interés (Gil 1982b, Gil y Martínez-Torregrosa 1983 y 1987, Driver y Oldham 1986, Burbules y Linn 1991, Gi1 et al. 1991, Wheatley 1991). Un esquema de esta estrategia de enseñanza queda reflejada en el cuadro II.

En las cuatro fases que esquematizan la estrategia de enseñanza propuesta en dicho cuadro, hemos intentado integrar aspectos esenciales que afectan la actividad científica y que han sido reiteradamente resaltados por la historia y filosofía de la ciencia, pero que a menudo no son suficientemente tenidos en cuenta en la enseñanza de las ciencias. Nos referimos concretamente a los problemas de contextualización del trabajo científico (reiaciones $\mathrm{C} / \mathrm{T} / \mathrm{S}$, toma de decisiones...) y a los componentes afectivos (interés por la tarea, clima de trabajo...).
Cuadro II

Estrategias de ensettanza para un aprendizaje como investigacion.

1. Plantear situaciones problemáticas que -teniendo en cuenta las ideas, vision del mundo, destrezas y actitudes de 108 alumnos y alumnas- generen interés y proporcionen una concepcion preliminar de la tarea.

2. Proponer a los estudiantes el estudio cualitativo de las situaciones problemáticas planteadas y la toma de decisiones, con la ayuda de las necesarias búsquedas bibliográficas, para acotar problemas precisos (ocasión para que comiencen a explicitar funcionalmente sus ideas).

3. Orientar el tratamiento científico de los problemas planteados, lo que conlleva, entre otros:

- La invencion de conceptos y emision de hipótesis (ocasión para que las ideas previas sean utilizadas para hacer predicciones).

- La elaboración de estrategias de resolución (incluyendo, en su caso, diserios experimentales) para la contrastación de las hipotesis a la luz del cuerpo de conocimientos de que se dispone.

- La resolución y el análisis de los resultados, cotejándolos con los obtenidos por otros grupos de alumnos y por la comunidad científica. Ello puede convertirse en ocasión de conflicto cognoscitivo entre distintas concepciones (tomadas todas ellas como hipotesis) y obligar a concebir nuevas hipotesis.

4. Plantear el manejo reiterado de los nuevos conocimientos en una variedad de situaciones para hacer gosible la profundizacion y aftanzamiento de los mismos, poniendo un énfasis especial en las relaciones ciencia/técnica/sociedad que enmarcan el desarro1 lo científico (propiciando, a este respecto, la toma de decisiones) y dirigiendo todo este tratamiento a mostrar el carácter de cuerpo coherente que tiene toda ciencia.

Favorecer, en particular, las actividades de sintesis (esquemas, memorias, mapas conceptuales...), la elaboración de productos (susceptibles de romper con plantearnientos excesivamente escolares y de reforzar el interés por la tarea) y la concepción de nuevos problemas.

El aprendizaje de las ciencias es concebido asíno como un simple cambio conceptual, sino como un cambio a la vez conceptual, metodológico y actitudinal.

Una estrategia de enseñanza como la propuesta puede calificarse de radicalmente constructivista, en el sentido de que contempla una participación efectiva de los alumnos en la construcción de los conocimientos y no la simple reconstrucción subjetiva de los conocimientos proporcionados por el profesor o los textos. Ello genera muy a menudo lógicas reticencias en el profesorado: ¿Hasta qué punto no se trata de una propuesta utópica? ¿Tiene sentido -se suele preguntar-esperar que los alumnos puedan construir por si solos todos los conoci. mientos que tanto tiempo y esfuerzos exigieron de los más relevantes científicos? ¿Cómo se concibe el trabajo en la clase? ¿Se trata de que los alumnos pasen la mayor parte del tiempo en el laboratorio (lo que supone olvidar las limitaciones del sistema educativo)? ¿Por qué no aceptar una pluralidad de enfoques, con momentos de investigación (de construcción de conocimientos) al lado de otros de recepción (a través de lecturas o de transmision verbal)? Todas estas cuestiones y muchas otras son planteadas por los profesores y los mismos 
investigadores, lo que muestra la necesidad de una mayor precisión en la presentación del modelo.

Dedicaremos el siguiente apartado a intentar contestar a dichas cuestiones, al tiempo que mostramos, de una forma más precisa, cómo concebimos esta orientación del aprendizaje de las ciencias como investigación. Pero queremos insistir, antes de referimos a nuestro enfoque particular, en que la idea de aproximar el trabajo de los alumnos a las características del trabajo científico atrae hoy un consenso creciente, fruto de la convergencia de Ios planteamientos constructivistas y de la profundización en las implicaciones de la historia y filosofia de las ciencias en la enseñanza de las ciencias (Hodson 1988, Astolfi y Develay 1989, Burbules y Linn 1991, Wheatley $1991 . .$.$) .$

Debemos advertir también que vamos a centramos en el nivel de la enseñanza secundaria superior, puesto que para alı̇mnos más jóvenes el objetivo no puede ser, en nuestra opinión, construir cuerpos de conocimientos científicos, sino una actividad exploratoria precientífica, de naturaleza muy diferente (Gil 1986, Astolfi y Develay 1989).

\section{EL APRENDIZAJE DE LAS CIENCIAS COMO INVESTIGACION}

La idea central del modelo de aprendizaje de las ciencias como investigación que proponemos consiste, como acabamos de ver, en el tratamiento de situaciones problemáticas abiertas de interés, a través de las cuales lo alumnos puedan participar en la construcción de los conocimientos. Una primera cuestión que ello plantea, recordemos, es la de hasta qué punto los alumnos pueden (re)construir unos conocimientos que tanto tiempo y esfuerzos han exigido a los científicos más notables.

\section{Los alumnos como investigadores noveles}

Es difícil no estar de acuerdo en que los alumnos por sí solos (?) no pueden construir todos (?) los conocimientos científícos. Como señala Pozo (1987), «es bien cierto que muchos de los conceptos centrales de la ciencia son bastante difíciles de descubrir para la mayor parte -si no para la totalidad- de los adolescentes e incluso de los adultos universitarios". Sin embargo, como trataremos de mostrar, de aquí no se sigue que se haya de recurrir necesariamente a la transmisión de dichos conocimientos ni que se haya de poner en cuestión las orientaciones constructivistas.

En efecto, es bien sabido que, cuando alguien se incorpora a un equipo de investigadores, puede alcanzar con relativa rapidez el nivel medio del resto del equipo. $Y$ ello no mediante una transmisión verbal, sino abordando problemas en los que quienes actúan de directores/ formadores son expertos.
La situación cambia, por supuesto, cuando se abordan problemas que son nuevos para todos. El avance, si lo hay, se hace entonces lento y sinuoso. La propuesta de organizar el aprendizaje de los alumnos como una construcción de conocimientos responde a la primera de las situaciones, es decir, a la de una investigación dirigida, en dominios perfectamente conocidos por el «director de investigaciones» (profesor) y en la que los resultados parciales, embrionarios, obtenidos por los alumnos, pueden ser reforzados, matizados o puestos en cuestion, por los obtenidos por los científicos que les han precedido. No se trata, pues, de «engañar» a los alumnos, de hacerles creer que los conocimientos se construyen con la aparente facilidad con que ellos los adquieren (Hodson 1985), sino de colocarles en una situación por la que los cientfficos habitualmente pasan durante su formación, y durante la que podrán familiarizarse mínimamente con lo que es el trabajo científico y sus resultados, replicando para elio investigaciones ya realizadas por otros, abordando, en definitiva, problemas conocidos por quienes dirigen su trabajo. El planteamiento constructivista del aprendizaje de las ciencias ha de responder a estas características de investigación dirigida. Un trabajo de investigación en el que constantemente se cotejan los resultados de los distintos equipos y se cuenta con la inestimable ayuda de un experto. No creemos necesario insistir aquí en los bien conocidos y documentados argumentos en favor del trabajo en pequeños grupos como forma de incrementar el nivel de participación y la creatividad necesaria para abordar situaciones no familiares y abiertas (Ausubel 1978, Solomon 1987, Linn 1987, Burbules y Linn 1991, Robinson y Niaz 1991), como indudablemente son las concebidas para posibilitar la construcción de conocimientos. Sí queremos insistir, por el contrario, en la necesidad, mucho menos tenida en cuenta, de favorecer la máxima interacción entre los grupos (Gil y Martínez-Torregrosa 1987, Gil et al. 1991, Wheatley 1991), a través de la cual los alumnos pueden asomarse a una característica fundamental del trabajo científico: la insuficiencia de las ideas y resultados obtenidos por un único colectivo y la necesidad de cotejarlos con los obtenidos por otros, hasta que se produzca suficiente evidencia convergente para que la comunidad científica los acepte. Nunca se insitirá bastante, en efecto, en que, por ejemplo, unos pocos resultados experimentales como los que se pueden obtener en un Iaboratorio escolar no permiten hablar de verificacion de hipótesis (Hodson 1985); de ahí la importancia de los intercambios intergrupos y la participación del profesor como «portavoz de otros muchos investigadores», es decir, de lo que la comunidad científica ha ido aceptando como resultado de un largo y difícil proceso. En este sentido, estamos totalmente de acuerdo con Pozo (1987) cuando afirma que «de lo que se trata es que el alumno construya su propia ciencia "subido a hombros de gigantes" y no de un modo autista, ajeno al propio progreso del conocimiento científico». No pensamos, sin embargo, que ello se favorezca con «la integración de la enseñanza por descubrimiento y de la enseñanza receptiva» (Pozo 1987), sinto mediante un trabajo colectivo de investigación dirigida, tan alejado del descubrimiento autónomo como de la transmisión de conocimientos ya elaborados (Gil 1983, Millar y Driver 1987). Dicho con otras palabras: entre la metáfora del alumno como simple receptor 
Cuadro ПI

Algunas concepciones erróneas sobre el trabajo científico que pueden ser transmitidas, explícita o implicitamente, por la enseñanza de las ciencias.

Visión empirista y ateórica: Se resalta el papel de la observación y de la experimentación «neutras» (no contaminadas por ideas apriorísticas»), olvidando el papel esencial de las hipótesis y de la construcción de un cuerpo coherente de conocimientos (teoria).

Por otra parte, pese a esta importancia dada (verbalmente) a la observación y experimentación, la enseñanza en generat, es puramente libresca, sin apenas trabajo experimental.

Se incide particularmente en esta visión atérica cuando se presenta el aprendizaje de la ciencia cono una cuestión de descubrimiento o se reduce a la práctica de los procesos con olviojo de los contenidos.

Visión rígida (algorítmica, «exacta», infalible...): Se presenta el «nıétodo científicon como conjunto de etapas a seguit mecánicamente. Se resalta, por otra parte, lo que supone rratamiento cuantitativo, control riguroso, etc., olvidando -o inciuso rechazando- todo lo que significa invención, creatividad, duda...

Visión aproblemática y ahistórica (ergo dogmática): Se transmiten conocimientos ya elaborados, sin mostrar cuáles fueron los problemas que generaron su construcción, cuál ha sido su evolución, las dificultades, etc. ni mucho menos aún, las limitaciones del conocimiento actual o las perspectivas abiertas.

Visión exclusivamente analítica, que resalta la necesaria parcialización de los estudios, su carácter acotado, simplificatorio, pero que olviđa los esfuerzos posteriores de unificación y de construcción de cuerpos coherentes de conocimientos cada vez más amplíos, el tratamiento de problemas «frontera» entre distintos dominios que pueden liegar a urirse, etc.

Vision acumulativa, lineal: Los conocimientos aparecen como fruto de un crecimiento lineal, ignorando las crisis, las remodelaciones profundas. Se ignora, en particular, la discontinuidad radical entre el tratamiento científico de los problemas y el pensamiento ordinario.

Visión de «sentido común»: Los conocimientos se presentan como claros, obvios, «de sentido común», olvidando que la construcción científica parte, precisamente, del cuestionamiento sistemático de to obvio.

Se contribuye implicitamente a esta visión cuando se practica el redaccionismo conceptual, es decir, cuando se presenta el paso de las concepciones alternativas de los alumnos a los conocimientos científicos como simple cambio de ideas, sin tener en cuenta los cambios metodolbgicos que exige dicha transformación, a saber, al ignorarse las diferencias substanciales que existen entre el pensamiento de sentido común y el tratamiento científico de los problemas.

Visión «yelada», elitista: Se esconde la significación de los conocinnientos tras el aparato matemático. No se hace un esfuerzo por hacer la ciencia accesible, por mostrar su carácter de construcción humana, en la que no faltan ni confusión ni errores... como los de los propios alumnos. En el mismo sentido, se presenta el trabajo científico como un dominio reservado a minorías especialmente dotadas, transmitiendo expectativas negativas hacia la mayoría de los alumnos, con claras discriminaciones de naniraleza social y sexual (la ciencia es presentada como una actividad eminentemente «masculina.»

Visión individualista: Los conocimientos cientificos aparecen como obra de genios aisłados, ignorándose el papel del trabajo colectivo, de los intercambios entre equipos... Se deja creer, en particular, que los resullados de un solo científico o equipo pueden verificar ofalsar una hipótesis.

Visión descontextualizada, socialmente neutra: Se olvidan las complejas relaciones C/T/S y se proporciona una imagen de los científicos como seres "por encima del bien y del mal», encerrados en torres de marfil y ajenos a las necesarias tomas de decisión. Cuando, en ocasiones, se tienen en cuenta las interacciones $C / T / S$, se suele caer en visiones simplistas: exaltación beata de la ciencia como factor absoluto de progreso o rechazo sistemático (a causa de su capacidad destructiva, efectos contaminantes, etc.).

y la que le asimila, siguiendo a Kelly, a un «investigador» autónomo (Pope y Gilbert 1983), proponemos la metáfora del «investigador novel» que integra además coherentemente las aportaciones de Vigotskii sobre la «zona de desarrollo potencial» y el papel del adulto en el aprendizaje. Las situaciones problemáticas abiertas, el trabajo científico en equipo y la interacción entre los equipos se convierten así en tres elementos esenciales de una orientación constructivista «radical» deI aprendizaje de las ciencias (Gil y Martínez-Torregrosa 87, Wheatley 1991).

\section{¿Actividad científica igual a trabajo experimental?}

Otra preocupación a menudo manifestada por buen número de profesores e investigadores es la de que esta orientación investigativa del aprendizaje parece primar eI trabajo de laboratorio (olvidando las limitaciones del sistema educativo) y dejar de lado actividades fundamentales como pueden ser la lectura o la escucha atenta y provechosa de una exposición: ¿Por qué -preguntanno aceptar una pluralidad de enfoques que incluya momentos de investigación junto a otros de recepción (a 
Figura I

Diagrama de un ciclo de investigación

Representación esquemática de un proceso colectivo extraordinariamente complejo.

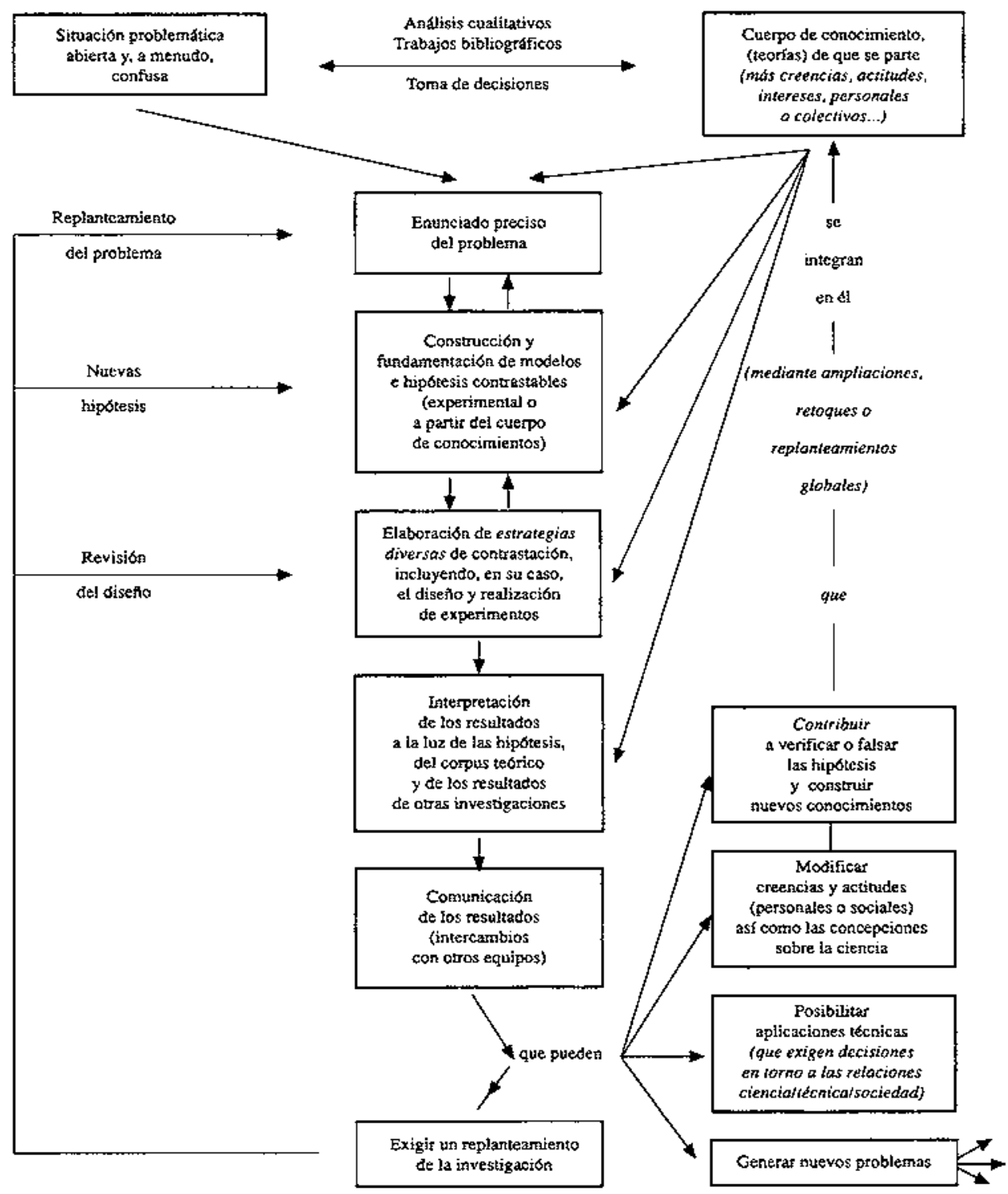

través de la lectura o de una buena conferencia)? ¡Alfin y al cabo mucho de lo que aprendemos proviene de la lectura!

Este tipo de comentarios merece una respuesta detenida, capaz de deshacer legítimas prevenciones frente a un supuesto «único camino» que rechace o ignore cualquier otra aportación. Para empezar, es necesario insistir contra las visiones reduccionistas del trabajo científico que estas mismas preguntas ponen en evidencia: ¿Cómo puede imaginarse que una estrategia investigativa deje de lado la lectura si más de la mitad del tiempo de un investigador se emplea en dicha actividad? ¿Y cómo suponer que la investigación se opone a escuchar a otros, si las presentaciones, debates, etc. constituyen una actividad regular dentro de cualquier equipo, además de una forma de comunicación privilegiada con el conjunto de la comunidad científica? Este «reduccionismo experimentalista» que asimila trabajo científico, casi exclusivamente, a trabajo de laboratorio es una de las muchas deformaciones que los profesores solemos transmitir sobre la naturaleza de la ciencia. En el cuadro III hemos intentado recoger algunas de las deformaciones más comunes, que proporcionan una imagen de la naturaleza 
de la ciencia en las antípodas de lo que la historia y filosofía de la ciencia nos muestra, pero extraordinariamente extendidas y difundidas, tanto por los media como por la misma enseñanza.

Resulta preciso transformar estas visiones reduccionistas y extremadamente simplistas del trabajo científico y aceptar su naturaleza de actividad abierta y compleja que incluye, como elementos clave, entre otros, Ia lectura o la comunicación. En la figura I presentamos un diagrama de un ciclo de investigación (Gil y Carrascosa 1992) que pretende salir al paso de dichas concepciones simplistas, tan difundidas entre el profesorado, sin cuya transformación no es posible modificar substancialmente la actividad en el aula (Bell y Pearson 1992).

Este reconocimiento de la importancia de actividades asociadas tradicionalmente al modelo de transmisión/ recepción no debe hacer pensar, sin embargo, en un ecléctico «todo vale». Desde una perspectiva de enseñanza/aprendizaje que se inspire en las estrategias del trabajo científico, leer un texto o escuchar al profesor no responden a la recepción de un conocimiento ya elaborado, sino que aparecen asociadas a, por ejemplo, una búsqueda bibliográfica destinada a precisar un problema - fundamentar una hipótesis, a la confrontación de la propia actividad con otros resultados o puntos de vista, etc. Se trata, pues, de actividades coherentes con lo que supone el tratamiento de situaciones problemáticas de interés y no, -insistimos-, de momentos de recepción que se combinan con otros de investigación.

Hay que subrayar, por otra parte, que una orientación como la que proponemos exige la transformación de las actividades fundamentales del aprendizaje de las ciencias -desde la introducción de conceptos al trabajo de laboratorio, pasando por la resolución de problemas de lápiz y papel... sin olvidar la evaluación- para que se conviertan en ocasión de construcción de conocimientos. Queremos insitir aquí, en particular, en que hablar de trabajo científico no significa necesariamente trabajo experimental: la resolución de problemas de lápiz y papel o la introducción đe conceptos exigen también, desde una óptica constructivista, una orientación investigativa. Nos remitimos, en lo que respecta a los problemas de lápiz y papel, a los numerosos artículos en los que hemos presentado su transformación en actividades de investigación y los resultados obtenidos (Gil y Martínez-Torregrosa 1983, Gil, Martínez-Torregrosa y Senent 1988, Gil, Dumas-Carré et al. 1990...). También hemos mostrado la posibilidad de asociar la introducción de conceptos al tratamiento de situaciones problemáticas que exigen aproximaciones cualitativas, invención de magnitudes operativas a título de hipótesis (cuya validez depende de la coherencia del cuerpo de conocimientos construido con su ayuda), etc. (Calatayud et al. 1990, Gil et al. 1991).

Esta transformación de las actividades «clásicas» no es, por supuesto, suficiente: no se puede concebir el aprendizaje como el resultado acumulativo de una serie de actividades disjuntas (introducción de conceptos, prácticas de laboratorio, resolución de problemas de lápiz y papel...). La construcción de cuerpos coherentes de co-
Cuadro IV

Aspectos a incluir en un currículo de ciencias para favorecer la construcción de conocimientos científicoss.

1. ¿Se presentan situaciones problemáticas abiertas (con objeto de que los alumnos puedan tomar decisiones para precisarlas)?

2. ¿Se plantea una reflexión sobre el posible interés de las situaciones propuestas que dé sentido a su estudio (considerando su relación con el programa general de trabajo adoptado, las posibles implicaciones $\mathrm{C} / \mathrm{T} / \mathrm{S}$, etc.)?

¿Se presta atención, en general, a potenciar las actitudes positivas y a que el trabajo se realice en un clima próximo a lo que es una investigación colectiva (situación en la que las opiniones, intereses, etc de cada individuo cuentan) y no en un clima de sometimiento a tareas impuestas por un profesor/ «capataz»?

3. ¿Se plantea un análisis cualitativo, significativo, que ayude a comprender y a acotar las situaciones planteadas (a la luz de los conocimientos disponibles, del interés del problema, etc.) y a formular preguntas operativas sobre lo que se busca?

4. ¿Se plantea la emisión de hipótesis fundamentadas, susceptibles de orientar el tratamiento de las situaciontes y de hacer explícitas, funcionalmente, las preconcepciones?

¿Se plantea, af menos, el manejo de alguna hipótesis?

¿Se presta atención a las preconcepciones?

5. ¿Se plantea la elaboración de estrategias (en plural), inçuyendo, en su caso, diseños experimentales?

¿Se pide, al menos, la evaluación crítica de algún diseño, etc.? ¿Se presta atención a la actividad práctica en sí misma (montajes, medidas...)?

6. ¿Se plantea el análisis detenido de Ios resuitados (su interpretación física, frabilidad, etc.), a lat luzdel cuerpo de conocimientos dispontble, de las hipótesis manejadas o de los resultados de otros autores?

¿Se plantea alguna reflexion sobre los posibles contlictos entre algunos resultados y las concepciones iniciales?

¿Se favorece la «autorregulación» del trabajo de los alumnos?

7. ¿Se plantea la consideración de posibles perspectivas (replanteamiento del estudio a otro nivel de complejidad, problemas derivados...)?

¿Se consideran, en particulat, las implicaciones $\mathrm{C} / \mathrm{T} / \mathrm{S}$ del estudio realizado (posibles aplicaciones, repercusiones negativas...)?

8. ¿Se pide un esfuerzo de integración que considere la contribución del estudio realizado a la construcción de un cuerpo coherente de conocimientos, las posibles implicaciones en otros campos de conocimientos, etc.?

¿Se pide algún trabajo de construcción de síntesis, mapas conceptuales, etc., que ponga en relación conocimientos diversos?

9. ¿Se plantea la elaboración de memorias científicas del trabajo realizado?

¿Se pide la lectura y comentario crítico de textos científicos? ¿Se presta atención a la verbalización, solicitando comentarios significativos que eviten el coperativismo mudon?

10. ¿Se potencia la dimensión colectiva del trabajo científico organizando equipos de trabajo y facilitando la interacción entre los equipos y la comunidad científica representada en la clase por el resto de los equipos, el cuerpo de conocimientos ya construido, los textos, el profesor como experto...)?

¿Se hace ver, en particular, que los resultados de una sola persona o de un solo equipo no pueden bastar para verificar o falscar una hipótesis?

¿Se plantea el manejo funcional del cuerpo de conocimientos aceptado por la comunidad (para ayudar, por ejemplo, a acotar una situación o analizar unos resultados, etc.)? 
nocimientos exige verdaderos programas de investigación capaces de orientar el trabajo de los alumnos. Abordaremos esta cuestión en el siguiente apartado.

\section{La transformación del curriculo en programas de actividades}

Como ya hemos señalado, la metáfora que guía nuestro modelo de enseñanza/aprendizajc de las ciencias concibe a los alumnos como «investigadores noveles» y al profesor como experto capaz de dirigir las investigaciones de los alumnos (quienes, de hecho, van a replicar trabajos bien conocidos por el profesor). Esta situación -muy alejada, claro está, de las investigaciones realizadas en dominios «frontera», desconocidos por la totalidad de los investigadores- permite evitar que los procesos resulten excesivamente erráticos y los aprendizajes inconexos, puesto que el profesor puede preparar verdaderos programas de investigación para orientar y prever el trabajo de los alumnos (Furió y Gil 1978, Gil 1982b, Gil y Martínez-Torregrosa 1987). Como Driver y Oldham (1986) han señalado, tal vez la implicación más importante del modelo constructivista en el diseño del currículo sea «no concebir el currículo como un conjunto de saberes y habilidades, sino como el programa de actividades a través de las cuales dichos saberes y habilidades pueden ser construidos y adquiridos».

Algunos profesores expresan el temor de que dichos programas puedan convertirse en una "camisa de fuerza». Ello es ciertamente posible... si las actividades no han sido correctamente concebidas para prever el desarrollo de la investigación. Pensemos que, después de todo, los alumnos van tan sólo a replicar investigaciones, es decir, a abordar situaciones nuevas y problemáticas para ellos pero bien conocidas por el profesor, lo que facilita una dirección flexible, sin impedir a los alumnos avanzar sus construcciones tentativas. Por supuesto, no existen recetas para garantizar que un determinado programa vaya a funcionar bien: su diseño constituye un trabajo de investigación aplicada para el profesorado (Driver y Oldham 1986), que debe someterse a control experimental (aplicación en el aula) y a la crítica de otros expertos. Desde este punto de vista, un programa de actividades aparece como algo siempre en (re)elaboración, sometido a retoques, añadidos y, en ocasiones, a completas remodelaciones.

Resulta particularmente importante cuidar de que el conjunto de actividades dé una visión correcta del trabajo científico, evitando los reduccionismos habituales (que hemos sintetizado en el cuadro III). A tal efecto hemos elaborado una parrilla de análisis para detectar errores y carencias en las que se puede incurrir al elaborar o llevar a la práctica los programas de actividades (Cuadro IV).

La utilización de los programas de actividades en la clase está presidida, como ya hemos dicho, por la idea de favorecer un trabajo colectivo, en el doble sentido de organizar la clase en pequeños grupos que abordan las actividades propuestas y de hacer posible los intercam- bios entre los grupos (Gil y Martínez-Torregrosa 1987, Wheatley 1981). Para favorecer esos intercambios y para poder proporcionar a los alumnos Ia dirección que precisa sis trabajo, la forma de organizar la actividad de Ios pequeños grupos no consiste en pedirles que aborden autónomamente el programa de actividades (solicitando la ayuda del profesor cuando la precisen) y organizar una puesta en común al final del trabajo. Esa forma de trabajo presenta, de acuerdo con nuestra experiencia, algunos inconvenientes, como son:

- Ruptura de la unidad de la clase a causa de los diferentes ritmos de trabajo de los grupos, lo que se traduce, cuando las tareas son extensas, en desfases difíciles de recuperar.

- Peligro de desorientación de los alumnos, dado que algunas actividades exigen la realización correcta de las preccdentes.

- Dificultad de que el profesor pueda satisfacer las peticiones de ayuda de los pequeños grupos (que la solicitan, a menudo, simultáneamente y sobre cuestiones diferentes).

Estas dificultades para el correcto seguimiento de la actividad de los alumnos nos han conducido a una forma más estructurada, consistente en realizar una puesta en común prácticamente después de cada actividad. Ello permite al profesor reformular y sintetizar las aportaciones, añadir información complementaria y orientar las actividades siguientes. Como es lógico, estas puestas en común no deben emplear, en general, mucho tiempo. Para conseguir esto empleamos técnicas diversas, como son la transcripción simultánea en la pizarra de las respuestas de los grupos, o una breve exposición de uno de los grupos que los otros equipos completan o critican si es necesario. Conviene añadir que no es preciso esperar a que todos los grupos hayan completado la tarea propuesta para pasar a la puesta en común, puesto que ello podría perjudicar el ritmo de la clase, provocando dispersiones e incluso aburrimiento. El profesor debe, pues, seguir con atención el trabajo de los grupos y pasar a la discusión en común en el momento oportuno. Esta puesta en común permitirá completar, en su caso, el trabajo de los grupos en retraso. Más aún: es posible que, en ocasiones, el trabajo de los grupos haya resultado ineficaz -tal vez porque la actividad no cstaba bien diseñada, to que obliga a su revisión-, o bien que los resultados constituyan aproximaciones correctas pero insuficientes y que el profesor deba reforzarlos añadiendo información, reformulăndolas, etc. Pero, como esta información y ayuda llega en respuesta a problemas que los grupos ya se han planteado, resulta significativa para los alumnos y es vista como algo funcional en el proceso de investigación seguido.

Señalemos, para terminar este apartado, que el desarrollo eficaz de un programa de investigación exige la transformación del clima đel aula para que se supere la habitual atmósfera de control autoritario, más típica de unos «trabajos forzados» que de una investigación científica (Gil et al. 1991, Tippins, Tobin y Hook, 1992). En esta transformación de las actividades y situaciones de 
aprendizaje, la historia y la filosofía de la ciencia aparecen de nuevo como recursos fundamentales, pudiendo ilustrar sobre las situaciones problemáticas enfrentadas por Ios científicos, las condiciones que pueden favorecer su tratamiento, etc.

\section{A MODO DE CONCLUSIÓN: LA DOCENCIA COMO INVESTIGACION}

La importancia de plantear el aprendizaje como tratamiento de situaciones problemáticas está siendo resaltada por diversos autores y aparece como una de las implicaciones fundamentales de las concepciones actuales sobre historia y filosofía de la ciencia (Burbules y Linn 1991, Wheatley 1991). Por otra parte, Ia orientación del aprendizaje de las materias científicas como una investigación con las características que acabamos de presentar es valorada sistemáticamente de forma muy positiva por los profesores que participan en seminarios y cursos dirigidos a introducir vivencialmente el nuevo modelo. En ocasiones, sin embargo, surge una cuestión que expresa una honda preocupación del profesorado: ¿Cómo exigir a un profesor o profesora todos los conocimientos que esta orientación de la enseñanza demanda? Pensemos, por ejemplo, en las dificultades que plantea la traducción del currículo en programas de actividades susceptibles de interesar a los alumnos y de favorecer un trabajo creativo y efectivo de construcción de conocimientos. Por supuesto que ningún profesor puede realizar una tarea de esta envergadura... pero es la propia pregunta la que carece de sentido. En efecto, cualquier estudio sobre metodología y epistemología de las ciencias revela unas exigencias para el trabajo científico al menos tan amplias y complejas como para el trabajo docente que estamos proponiendo; pero a ningún científico se le exige que posea el conjunto de saberes y destrezas necesarios para el tratamiento científico de los proble-

\section{REFERENCIAS BIBLIOGRÁFICAS}

AGABRA, J., ASTOLFI, J.P., et al. (Equipe de Recherche ASTER), 1985. Procédures d'apprentissage en Sciences Expérimentales. (INRP: París).

ANDERSON, C.W. y SMITH, E.L., 1983. Childrens' conceptions of light and color. Developing the concept of unseen rays. Annual meeting of the American Educational Research Association, Montreal.

ASTOLFI, J.P. y DEVELAY, M., 1989. La Didactique des Sciences. (PUF: París). mas: se tiene muy claro que se trata de una tarea colectiva. Del mismo modo, el trabajo docente tampoco es o, mejor dicho, no debería ser una tarea aislada, y ningún profesor/a ha de sentirse oprimido por un conjunto de saberes que, con toda seguridad, sobrepasan las posibilidades de un ser humano. Lo esencial es que pueda darse un trabajo colectivo en todo el proceso de enseñanza/ aprendizaje: desde la preparación de las clases a la evaluación.

Con este punto de vista, la complejidad de la actividad docente deja de verse como un obstáculo a la eficacia y un factor de desánimo, para convertirse en una invitación a romper con la inercia de una enseñanza monótona y sin perspectivas y a aprovechar la enorme creatividad potencial de la nueva orientación de la docencia. De este modo, el problema de falta de interés por la docencia de muchos profesores -que se está traduciendo en numerosos países en una grave carencia de profesores de ciencias- puede encontrar una vía de solución similar a la que se contempla para los propios alumnos: implicación en tareas abiertas, creativas, en la difusión de experiencias innovadoras, en los intercambios entre grupos de profesores; en definitiva: en un trabajo colectivo de innovación e investigación que puede transmitir a la docencia, al igual que al aprendizaje, todo el interés de la actividad científica. Ello implica todoun programa de (auto)formación de Ios docentes que no podemos desarrollar aquí -por obvias razones de espacio-, pero que supone un elemento absolutamente esencial de este modelo de aprendizaje de las ciencias como actividad de investigación.

\section{NOTA}

1 Una primera versión de este trabajo fue presentada como conferencia invitada en la «International Conference on History of the Physical-Mathematical Sciences and the teaching of Sciences» (Madrid, septiembre de 1992).
ASTOLFI, J.P., VERIN, A. et al. (Equipe de Recherche ASTER), 1985. Formation Scientifique et travail autonome. (INRP: París).

AUSUBEL, D.P., 1968. Educational Psychology. A cognitive View. (Holt, Rineheart and Winston, Inc: Nueva York).

BACHELARD, G., 1938. La formation de l'esprit scientifique. (Vrin: París).

BELL, B.F. y PEARSON, J., 1992. International Journal of Science Education, 14(3), pp. 349-361. 
BURBULES, N. y LINN, M., 1991. Science education and philosophy of science: congruence or contradiction?, International Journal of Science Education, 13 (3), pp. 227241.

CALATAYUD, M.L., CARRASCOSA, J., GIL, D. et al. 1990. La construcción de las ciencias fisico-quimicas (Programas gulas de trabajo y comentarios para el profesor). (Nau Llibres: Valencia).

CARRASCOSA, J. y GIL, D, 1982. Los errores conceptuales en la enseñanza de la física $I$. Un estudio de su persistencia. Actas de las Primeras Jornadas de Investigación Didactica en Física y Química. (ICE de la Universitat de Valencia).

CARRASCOSA, J, y GIL, D., 1985. La metodologia de la superficialitat $i l^{\prime}$ aprenentatge de les ciencies, Enseñanza de las Ciencias, 3 (2), pp. 113-120.

CHAMPAGNE, A.B., GUNSTONE, R.F. y KLOPFER, L.E., 1985. Effecting changes in cognitive structures among physics students, en West, L.H.T. y Pines, A.L. (eds), Cognitive structure and conceptual change. (Acadernic Press: Orlando. FL).

CLEMENT, J., 1983. A conceptual model discussed by Galileo and intuitivily used by physics students, en Genter, D. y Stevens, A.L. (eds.), Mental Models (Erlbaum, Hilsdalle: Nueva York).

CLEMINSON, A., 1991. Establishing an epistemological base for science teaching in the light of contemporary notions of the nature of science and of how children learn science, Journal of Research in Science Teaching, 27 (5), pp. 429445.

CLOSSET, J.L., 1983. D'ou proviennent certaines erreurs rencontrées chez les élèves et les étudiants en électrocinetique...?, Bulletin de l'Union des physiciens, 657 , pp. 81-102.

COBB, P., WOOD, T. y YACKEL, E., 1991. Analogies from the philosophy and sociology of science for understanding classroom life, Science Education, 75 (1), pp. 23-44.

COLL, C., 1988. Conocimiento psicologico y práctica educativa. (Barcanova: Barcelona).

DISESSA, A.A., 1982. Unleaming Aristotelian Physics: a study of Knowledge-based learning, Cognitive Science, 6 , pp. $37-75$.

DRIVER, R. y OLDHAM, V., 1986. A constructivist approach to curriculum development in science, Studies in Science Education, 13, pp. 105-122.

DUSCHL, R. y GITOMER, D., 1991. Epistemological Perspectives on conceptual change: implications foreducational practice, Journal of Research in Science Teaching, 28 (9), pp. $839-858$.

ENGEL, E. y DRIVER, R., 1986. A study of consistency in the use of students' conceptual frameworks across different task contexts, Science Education, 70 (4), pp. 473-496.

ESCUDERO, T., 1985. Las actitudes en la enseñanza de las ciencias: un panorama complejo, Revista de Educación, 778 , pp. $5-25$

FREDETTE, N. y LOCHHEAD, J., 1981. Students conceptions of electric current, The Physics Teacher, Vol. 18, pp. 194198.

FURIÓ, C., HERNÁNDEZ, J, y HARRIS, H., 1987. ParalleIs between adolescents' conception of gases and the history of Chemistry, Journal of Chemical Education, 64 (7), pp. 617618.
GIL, D., 1982a. L'ensenyament de les ciències a tes Escoles de Magisteri. Primeres Jomades de Recerca Educativa. Lleida. (ICE Universitat Autònoma: Barcelona), pp. 315-323.

GIL, D., 1982b. La investigación en el aula de Física y Química. (Anaya: Madrid).

GIL, D., 1983. Tres paradigmas básicos en la enseñanza de las ciencias, Enseñanza de las Ciencias, 1 (1), pp. 26-33.

GL, D., 1986. La metodología científica y la enseñanza de las ciencias: unas relaciones controvertidas, Enseñanza de las Ciencias, 4(2), pp. $111 * 121$.

GIL, D. y CARRASCOSA, J., 1985. Science leaming as a conceptual and methodological change, European Journal of Science Education, 7 (3), pp. 231-236.

GIL, D. y CARRASCOSA, J., 1990. What to do about science misconceptions?, Science Education, 74 (4).

GIL, D. y CARRASCOSA, J., 1992. Approaching pupils' learning to scientific construction of knowledge: some implications of the History and Philosophy of Science in Science Teaching, Proceedings of the Second International Conference on History and Philosophy of Science in Science Teaching. (Kingston, Ontario, Canada), pp. 375-389.

GIL, D., CARRASCOSA, J., FURIO, C. y MARTÍNEZTORREGROSA, J., 1991. La enseñanza de las ciencias en la educación secundaria. (Horsori: Barcelona).

GIL, D., DUMAS-CARRÉ, A. et al. Paper and pencil problem solving in the physical sciences as an activity of research, Studies in Science Education, 18, pp. 137-151.

GIL, D. y MARTÍNEZ-TORREGROSA, J., 1983. A model for problem-solving in accordance with scientific methodology, European J. of Science Education, 5(4), pp. 447-455.

GIL, D. y MARTÍNEZ-TORREGROSA,J., 1987. Los programasguía de actividades: una concreción del modelo constructivista de aprendizaje de las ciencias, Investigación en la Escuela, 3, pp. 3-12.

GIL, D., MARTÍNEZ-TORREGROSA, J. y SENENT, F., 1988. El fracaso en ia resolución de problemas: una investigación orientada por nuevos supuestos, Enseñanza de las Ciencias, 6(2), pp. 131-146.

GIORDAN, A., 1978. Observation-Experimentation: mais comment les êlèves apprennent-ils?, Revue Francaise de Pedagogie, 44, pp. 66-73.

GIORDAN, A., 1989. De las concepciones de tos alumnos a un modelo de aprendizaje alostérico, Investigación en la Escuela, 8, pp. 3-14.

GRUENDER, C.D. y TOBIN, K., 1991. Promise and Prospect, Science Education, 75 (1), pp. 1-8.

HALLOUN, I.A. y HESTENES, D., 1985. Common sense concepts aboutmotion, American Journal of Science Education, 7 (3), pp. 231-236.

HASHWEH, M.Z., 1986. Towards an explanation of conceptual change, European Journal of Science Education, 8 (3), pp. 229-249.

HEWSON, P.W., 1985. Epistemological commitments in the learning of science: examples from dynamics, European Journal of Science Education, 8 (2), pp. 157-171.

HEWSON, M.G. y HEWSON, P.W., 1984. Effect of instruction using students prior knowledge and conceptual strategies on science learning, European Journal of Science Education, 6 (1), pp. 1-6. 
HEWSON, P.W. y HEWSON, M.G., 1988. On appropriate conception of teaching science: a view from studies of science leaming, Science Education, 72 (5), pp. 597-614.

HEWSON, P.W. y THORLEY, N.R., 1989. The conditions of conceptual change, International Journal Science Education, Vol 11, special issue, pp. 541-553.

HILLS, G.L., 1989. Students «untutored» beliefs about natural phenomena: primitive science or commonsense?, Science Education, 73(2), pp. 155-186.

HODSON, D., 1985. Philosophy of science, science, and science education, Studies in Science Education, 12, pp. 25-57.

HODSON, D., 1988. Towards a phylosophically more valid science curriculum, Science Education, 72 (1), pp. 19.40.

HODSON, D., 1992, Assessment of practical work. Some considerations in philosophy of Science, Science and Education, 1(2), pp. 115-144.

HOLTON, G. y ROLLER, D., 1963. Fundamentos de la Física Moderna. (Reverté: Barcelona).

HOST, V., 1978. Procédures d'aprentissage spontanées dans la formation du scientifique, Revue Francaise de Pédagogie, 45, pp. $103-110$.

IZQUIERDO, M., 1992. Reconsidering the sciences curriculum starting from contemporary (converging) models of science and cognition: a research program. Proceedings of the Second International Conference on History and Philosophy of Science in Science Teaching. (Kingston, Canadá), pp. 517. 529.

JAMES, R.K. y SMITH, S., 1985. Alienation of students from science in grades 4-12, Science Education, 69, pp. 39-45.

KING, B., 1991. Beginning teachers' knowledge of and attitudes towards History and Philosophy of science, Science Education, $75(1)$, pp. $135-141$.

KOYRE, A., 1981. Estudios galileanos. (Siglo XXI: México)

KUHN, T.S., 1971. La estructura de las revoluciones cientificas. (Fondo de cultura económica; México).

LANGEVIN, P., 1926. La valeur éducative de l'histoire des sciences, Bulletin de la Societé Francaise de Pedagogie, 22 , diciembre de 1926.

LAUDAN, L., 1984. Science and values: the aims of science and their role in scientific debate. (University of California Press: Berkeley).

LEBOUTET, L., 1973. L'enseignement de la Physique. (PUF: París).

LINN, M., 1987. Establising a research base for science education: challenges, trends and recomendations, Journal of Research in Science Teaching, 24(3), pp. 191-216.

LORSBACH, A.W., TOBIN, K., BRISCOE, C. y LAMASTER, S.V., 1992. An interpretation of assessment methods in middie school science, International Journal of Science Education, 14(3), pp. 305-317.

MATTHEWS, M.R., 1990. History, Philosophy and Science Teaching: A Rapprochement, Studies in Science Education, 18, pp. 25-51.

MCDERMOTT,L.C., 1984. Research on conceptual understanding in mechanics, Physics Today, julio, pp. 24-34.

MILLAR, R. y DRIVER, R., 1987. Beyond processes, Studies in Science Education, 14, pp. 33-62.
MINISTRELL, J., 1982. Explaining the «at rest» condition of an object, Physics Teacher, 20, pp. 10-14.

MINISTRELL, J., 1984. Teaching for the development of understanding of ideas: forces on moving objects, en Anderson C.W. (ed.), Observing science classroom: observing science perspectives from research and practice. (ERIC/SMAC: Columbus, $\mathrm{OH}$ ).

MORENO, M., 1986. Ciencia y construcción del pensamiento, Enseñanza de las Ciencias, 4(1), pp. 57-64.

NAVARRO, V., 1983. La Historia de las ciencias y la enseñanza, Enseñanza de las Ciencias, $1(1)$, pp. 50-53.

NOVAK, J.D., 1979. The reception learning paradigm, Journal of Research in Science Teaching, 16, pp. 481-488.

NOVAK, J.D., 1988. Constructivismo humano: un consenso emergente, Enseñanza de las Ciencias, 6 (3), pp. 213-223.

NUSBAUM, J. y NOVICK, S., 1982. Alternative frameworks, conceptual conflict and accommodation: towards a principled teaching strategy, Instructional Science, 11, pp. 183-200.

OSBORNE, R. y FREYBERG, P., 1985. Learning in Science. (Heinemann: Postmouth, NH).

OSBORNE, R. y WITTROCK, M., 1983. Leaming Science: a generative proceess, Science Education, 67, pp. 490-508.

OSBORNE, R. y WITTROK, M., 1985. The generative leaming model and its implications for science education, Studies in Science Education, 12, pp. 59-87.

PIAGET, J., 1970. La epistemología genética. (Redondo: Barcelona).

POPE, M.L. y GILBERT, J., 1983. Personal experience and the construction of knowledge in science, Science Education, 67 , pp. 193-203.

POSNER, G. J. et al., 1982. Accomodation of a scientific conception: towards a theory of conceptual change, Science Education, 66, pp. 211-227.

POZO, I.I., 1987. Aprendizaje de la ciencia y pensamiento causal. (Visor: Madrid).

POZO, J.I., 1989. Teorías cognitivas del aprendizaje. (Morata: Madrid).

RESNICK, L.B., 1983. Mathematics and Science Learning: a new conception, Science, 220, pp. 477-478.

REVE, R. et al., 1987. Everyday and academic thinking: implications for learning and problem solving, Journal of Curriculum Studies, 19(2), pp. 123-133.

ROBINSON, W.R. y NIAZ, M., 1991. Performance based on instruction by lecture or by interaction and its relationship to cognitive variables, International Journal of Science Education, $13(2)$, pp. $203-215$.

ROTH, K.J., 1984. Using classroom observations to improve science teaching and curriculum materials, en Anderson C.W. (ed.), Observing science classrooms: observing science perspectives from research and practice (ERIC/SMEÁC,OH).

SALTIEL, E. y VIENNOT, L., 1985. ¿Qué aprendemos de las semejanzas entre las ideas históricas y el razonamiento espontáneo de los estudiantes?, Enseñanza de las Ciencias, 3 (2), pp. 137-144.

SÁNCHEZ RON, J.M., 1988. Usos y abusos de la historia de la Física en la Enseñanza, Enseñanza de las Ciencias, pp. $179 \cdot 188$. 
SCHIBECI, R.A., 1984. Attitudes to science: an update, Studies in Science Education, 11, pp. 26-59.

SEQUEIRA, M. y LEITE, L., 1991. Aitemative conceptions and history of science in physics teacher education, Science Education, 75 (1), pp. 45- 56.

SHUELL, T.J., 1987. Cognitive psychology and conceptual change: implications for teaching science, Science Education, 71 (2), pp. 239-250.

SOLOMON, J., 1987. Social influences on the construction of pupils' understanding of science, Studies in Science Education, 14, pp. 63-82.

SOLOMON, J., 1991. Teaching about the nature of science in the British National Curriculum, Science Education, 75 (1), pp. 95-103.

TIPPINS, D., TOBIN, K. y HOOK, K., 1992. Ethicai decisions at the heart of science teaching: reframing assessment dilemmas from a constructivist perspective. Proceedings of the Second International Conference on History and Philosophy of Science in Science Education (Kingston, Canada), pp. 513-526.

VIENNOT, L., 1989. Obstacle epistémologique et raisonnement en physique. Tendence au contoumement des conflicts chez les enseignants, en Construction des savoirs: obstacles et conflits. (Agence d'ARC Inc: Ottawa).

WHEATLEY, G.H., 1991. Constructivist perspectives on Science and Mathematics learning, Science Education, $75(\mathrm{I})$, pp. 9.21.

WHITAKER, R.J, 1983. Aristotle is not dead: student understanding of trajectory motion, American Journal of Physics, 51, pp. 352-357.

WHITE, T.R. y GUNSTONE, F.R., 1989. Metalearning and conceptual change, International Journal Science Education, 1 I. pp. 577-586.

YAGER, R.E. y PENICK, J.E., 1983. Analysis of the current problems with school science in the USA, European Journal of Science Education, 5, pp. 459-463.

YAGER, R.E. y PENICK, J.E., 1986. Perception of four groups towards science classes, teachers and value of science, Science Education, 70 (4), pp. 335-363.

ZIETSMAN, A.I. y HEWSON,P.W., 1986. Effects of instruction using microcomputer simulations and conceptual change strategies on science leaming, Journal of Research in Science Teaching, 23(1), pp. 27-93. 